\title{
EL TERREMOTO DE 1746 Y SU IMPACTO EN LA SALUD EN LA CIUDAD DE LIMA
}

\author{
Carlos Carcelén (101,a, Daniel Morán (101,b, Laura Amador (ib)1,c \\ 1 Universidad San Ignacio de Loyola, Lima, Perú. \\ a Magíster en Historia de América; ${ }^{\mathrm{b}}$ doctor en Historia; ${ }^{\mathrm{c}}$ bachiller en historia.
}

\section{RESUMEN}

Durante el siglo XVIII la ciudad de Lima fue afectada por una serie de desastres de origen natural y de epidemias que mermaron tanto la población como la producción agrícola. A continuación, analizaremos el caso del terremoto producido en Lima el 28 de octubre de 1746 y su impacto en el sistema de salud de la ciudad. Dada la magnitud de su destrucción en la infraestructura y el alto número de muertes, marcó un hito en la sociedad limeña de la época.

Palabras Claves: Terremotos; Epidemias; Perú (fuente: DeCS BIREME).

\section{THE EARTHQUAKE OF 1746 AND ITS IMPACT ON HEALTH IN THE CITY OF LIMA}

\begin{abstract}
During the 18th century, the city of Lima was affected by a series of natural disasters and epidemics that depleted both the population and agricultural production. Next, we will analyze the case of the earthquake in Lima on October 28, 1746 and its impact on the city's health system, given the magnitude of the destruction of infrastructure and the high number of deaths it marked a milestone in Limas society at the time.
\end{abstract}

Keywords: Earthquakes; Epidemics; Peru (source: MeSH NLM).

\section{TERREMOTO, DESTRUCCIÓN Y EPIDEMIA EN 1746}

Hasta antes de que el terremoto ocurrido en 1746 destruyese Lima y Callao, en la capital virreinal se registraron catorce terremotos en los años 1582, 1586, 1609, 1630, 1655, 1687, 1694, $1699,1716,1725,1735,1734$ y 1743 , siendo el de 1746 el más destructivo ${ }^{(1)}$. Se puede observar la constante destrucción de la infraestructura hidráulica de Lima y su entorno productivo originado por una serie de sucesivos terremotos, que son resumidos en la investigación de María Eugenia Petit-Breuilh Sepúlveda para toda la época colonial ${ }^{(2)}$ y en el catálogo publicado por Lizardo Seiner Lizárraga ${ }^{(3)}$ para los siglos XV al XVIII.

El 28 de octubre de 1746 a las 10:30 de la noche ${ }^{(4)}$, Lima fue sacudida por un terremoto que dejó unas 1300 personas muertas ${ }^{(5)}$ mientras que en El Callao el número fue mayor, unos 3800 muertos $^{(2)}$, ya que el sismo fue seguido por un tsunami, con olas de entre 10 y 24 metros ${ }^{(6)}$. En total se puede hablar de la pérdida del $8 \%$ de la población total de Lima y Callao, que se calculaba en unos 65000 habitantes en esos años ${ }^{(4)}$. Se calcula la fuerza sísmica en $8.4^{\circ}$ en la escala

Citar como: Carcelén C, Morán D, Amador L. El terremoto de 1746 y su impacto en la salud en la ciudad de Lima. Rev Peru Med Exp Salud Publica. 2020;37(1):16468. Doi: https://doi.org/10.17843/ rpmesp.2020.371.4547

Correspondencia: Carlos Carcelén; carlos.carcelen@usil.pe

Recibido: $19 / 05 / 2019$ Aprobado: 22/01/2020 En línea: $23 / 03 / 2020$
Richter. La severidad del terremoto fue tal, que la intensidad en la escala Mercalli Modificada se ha estimado en X-XI, lo que indica un grado de destrucción altísimo ${ }^{(1)}$.

Respecto a los daños se requirió la reconstrucción de «las fortificaciones y población de El Callao, al igual que los dos edificios más significativos de la capital: el palacio virreinal y la catedral. Los principales hospitales, conventos y templos sufrieron derrumbes y grandes destrozos, únicamente permaneció ilesa la iglesia de San Francisco y la de Santo Domingo sufrió daños menores» ${ }^{(7)}$.

Pérez-Mallaína ${ }^{(3)}$, usando los testimonios de Eusebio Llano Zapata y del arzobispo de Lima Pedro Antonio Barroeta, describe la tragedia de las epidemias posteriores al terremoto: "Como es natural, el hacinamiento, la carencia de higiene y la exposición a las inclemencias del tiempo y el hambre, 'puerta franca de las pestes y llave maestra de las enfermedades', terminaron por desencadenar epidemias, entre ellas las de 'tercianas', los 'dolores pleuríticos', los 
'efluvios de vientre' y el temible 'tabardillo', es decir el tifus».

Algunos testimonios de recién llegados mostraban sorpresa al ver a los leprosos transitar por las calles y al asentarse poblaciones sobre los estercoleros, en habitaciones separadas por una simple estera, los contagios eran rápidos y mortales. Tras el terremoto las epidemias y mortandad se presentaron pese a los esfuerzos de las autoridades, en especial del Virrey, por restablecer el ritmo del abastecimiento de alimentos, tanto los traídos desde Chile, como el trigo, o del interior, como la carne, los frutos y la nieve de Huarochirí. Asimismo, otra prioridad fue despejar las cañerías y restablecer el flujo de agua en la ciudad, por las necesidades sanitarias y alimenticias, y por las de energía hidráulica para los molinos de trigo ${ }^{(5)}$.

Luego del movimiento inicial, la gente se volcó a las calles. El virrey Manso de Velasco ${ }^{(8)}$ narra que debió retornar a la Plaza Mayor poco después del terremoto, la cual se encontraba llena «... de innumerable gente, que, poseída del susto, solo pensaba en buscar un lugar que no pudiese serle de sepulcro; y el resto del Pueblo, se hallaba alojado en otras plazas, en Huertas, y Campañas». Y en dicha situación permanecieron los sobrevivientes por varias semanas. Los más pobres se asentaron en las plazas o se dirigieron al campo, y fueron víctimas de las enfermedades respiratorias o infecciosas generadas por la exposición a los elementos de la naturaleza y las precarias condiciones sanitarias.

Los cadáveres se encontraban a la intemperie por lo que las autoridades se vieron en la necesidad de abrir fosas en las plazas y calles. Aunque el trabajo de sepultar tal cantidad de cuerpos era una tarea percibida como peligrosa debido las condiciones y se temía la aparición de epidemias debido a la gran cantidad de restos, Manuel de Odriozola recoge que se calcularon «más de tres mil mulas y caballos podridos debajo de las ruinas, sin haber sido posible sacarlos. Al cuarto día de ocurrido el terremoto se continuó enterrando los cuerpos en fosas excavadas en los cementerios y plazas llegando según testimonios de la época a 1300» ${ }^{(9)}$.

No obstante, el virrey convocó a la Cofradía de la Caridad, la cual, con la asistencia de quienes se encargaban de la limpieza de las calles, se encargó de llevar los cadáveres a las iglesias para librar a la ciudad de la infección con que estaba amenazada. A propósito de esta tarea, el jesuita Pedro Lozano señala que dichas labores ocasionaron la muerte de quienes las practicaron, según la creencia de la época, a causa del olor de los cadáveres. Además de la falta de condiciones adecuadas para la remoción de escombros y cuerpos «y no habiendo quedado en pie siquiera un granero, o pósito de las cosas necesarias a la vida» ${ }^{(9)}$.

Los «miasmas» eran considerados la principal causa de contagio de las enfermedades ${ }^{(10)}$, y su propagación era un temor que crecía cada vez más debido a las condiciones sanitarias en que se encontraba la población. Generalmente los miasmas eran entendidos como un efluvio que se desprendía de las aguas estancadas, los cuerpos enfermos o en descomposición, incluso las casonas coloniales tenían un segundo patio en la parte posterior $y$, «en dirección opuesta al viento, los cuartos de los esclavos, para que el aire se llevase supuestos malos olores» ${ }^{(11)}$.

Ante la amenaza de una epidemia, se recogieron los muertos para darles sepultura en grandes zanjas que se abrieron en las plazas. En estas condiciones, «en que el vecindario se hallaba a la intemperie, fuertes vientos y tenaces aguaceros -desconocidos en la costa- acentuaron los padecimientos de la atribulada población, y cerca de 2000 personas fallecieron como consecuencia de afecciones bronquiales, disentería y enfermedades gastrointestinales. En los campos enjambres de sabandijas asolaron los sembríos» ${ }^{(12)}$.

Sobre las epidemias Lastres ${ }^{(13)}$ señala que, en 1746 , luego del «el gran terremoto",, hubo una epidemia de tabardillo (tifus), que ocasionó la muerte de entre seis y ocho mil personas. Aunque encontramos una cifra más modesta en los Anales del $\mathrm{Cuzco}^{(14)}$ donde se menciona que tras el terremoto perecieron en Lima «más de dos mil personas, víctimas del tabardillo, pleuresías y distintas enfermedades que tomaron forma de epidemia», lo que sí es concluyente es que la población vio afectada su salud seriamente afectada debido a las condiciones sanitarias tras el terremoto. Las enfermedades eran transmitidas por vectores que surgieron a raíz de los cambios climáticos generados tras el terremoto y tsunami, en principio y, posteriormente, el evento del El Niño de 1747. Además, existieron enfermedades originadas por el agua acumulada tras las inundaciones; la contaminación por agua propició brotes de tifoidea.

\section{IMPACTO EN LA SALUD}

Entre las décadas de los cincuenta y los sesenta, las responsabilidades del sector sanitario fueron variando, enfocándose no solo en aquellos que acudían en busca de atención médica, sino también en los que no lo hacían, cambiar «el curar» por el «hacerse cargo de la población», coordinando lo social con lo clínico ${ }^{(15,16)}$. Evidentemente, para 1746 aún no se entendía en su totalidad el concepto de salud pública y la ciudad se recomponía gradualmente luego de numerosas actividades religiosas sin que se establezca una discusión sobre cómo mejorar las condiciones existentes ante el próximo terremoto. En las siguientes líneas se mencionan los cambios en la respuesta de las autoridades ante estos hechos siendo posible notar un incipiente interés por mejorar las condiciones de vida de la población pese a que los primeros visos de salud pública llegarían tras la ilustración.

La población sobreviviente se desplazó hacia las zonas rurales y refugios temporales lo cual incrementó el riesgo de infecciones. Las principales enfermedades que padecen las 
personas agrupadas en espacios insuficientes son las diarreas y la disentería, tos ferina, tuberculosis, sarna y otras dermatosis ${ }^{(17)}$, se trata de un patrón epidemiológico que se repite luego de un desastre natural y que se dio en esta población, de acuerdo a los testimonios que refieren un mayor número de víctimas por causas indirectas que por el desastre mismo.

La transmisión de enfermedades contagiosas inmediatamente a desastres naturales puede ser influida por seis factores: a) las enfermedades existentes en la población antes del desastre y los niveles endémicos que esta padecía; b) los cambios ecológicos provocados por el desastre; c) los desplazamientos; $\mathrm{d}$ ) el daño a edificios de servicios públicos; e) el desquiciamiento de los programas de control de enfermedades y f) la alteración de la resistencia individual a las enfermedades ${ }^{(17-20)}$. En el caso del terremoto de Lima se cumplieron casi todas las condiciones.

Si bien, la ciudad contaba hasta con catorce hospitales, como se observa en la Tabla 1, estos no contaban con el presupuesto apropiado para satisfacer las necesidades sanitarias de la población. De un presupuesto total de 1882701 pesos correspondientes a los primeros años del gobierno del conde de Superunda, se destinaban a ayudar al mantenimiento de los hospitales para blancos, indios y castas, 27998 pesos, es decir, el $1,5 \%{ }^{(5)}$.

Luego del terremoto, gran parte de los hospitales quedaron destruidos por lo que su reconstrucción era urgente ${ }^{(10)}$.
En el Real Hospital San Andrés, de españoles y sus hijos, de 2449 ingresos tras el terremoto y hasta un año después murieron 290. En el de Santa Ana, para indios e indias, de 2120, murieron 422 , sin contar los 42 que murieron sepultados la noche del terremoto. En el hospital de San Bartolomé de 202 murieron $75^{(9)}$; igualmente, el Hospital del Callao, que auxiliaba a la «gente de mar y tropa», se destruyó como consecuencia del terremoto, por lo que los enfermos debían concurrir a Lima para ser atendidos, en consecuencia, hubo más muertes indirectas por la demora en el traslado ${ }^{(13)}$.

La escasez de alimentos, la destrucción de la infraestructura hidráulica de la ciudad y de la zona agrícola aledaña, que produjo una mayor escasez de agua, y el incremento de la temperatura y la humedad por ser el inicio del verano posibilitaron el desarrollo de una serie de epidemias, como el tifus, la sarampión, las infecciones respiratorias y gastrointestinales, que costaron la vida de 2000 habitantes de la zona de desastre desde noviembre de 1746 hasta febrero de $1747^{(21)}$.

En los meses previos al terremoto, por un cálculo elaborado sobre los padrones de confesión, «se regularon de población 60000 almas, incluso los campos y haciendas», el evento sísmico, junto a las epidemias subsiguientes disminuyeron la población en seis u ocho mil individuos ${ }^{(22)}$; para 1755 el crecimiento vegetativo no había logrado cubrir la cifra de desaparecidos ${ }^{(21)}$.

Tabla 1. Hospitales públicos y religiosos (siglo XVIII)

\begin{tabular}{|c|c|c|}
\hline Hospital & Tipo & Objetivo \\
\hline Hospital Real de San Andrés & Público & $\begin{array}{l}\text { Con capacidad para } 200 \text { camas donde eran atendidos en salas separadas españoles, } \\
\text { negros y mulatos pobres. Con salas para enfermedades cotidianas, contagiosas y } \\
\text { para enfermos mentales. }\end{array}$ \\
\hline Hospital de San Pedro & Público & Fundado por el clérigo Francisco de Molina para eclesiásticos pobres. \\
\hline Hospital del Espíritu Santo & Público & $\begin{array}{l}\text { Localizado en el Callao para gente de mar y tropa. Con } 70 \text { camas para los marine- } \\
\text { ros enfermos que legaban al puerto. A cargo de los religiosos de San Juan de Dios. }\end{array}$ \\
\hline Hospital de San Bartolomé & Público & Para albergar a negros. \\
\hline Hospital de Santa Ana & Público & $\begin{array}{l}\text { Fundado por el arzobispo Jerónimo de Loayza. Contaba con } 300 \text { camas para in- } \\
\text { dios e indias. }\end{array}$ \\
\hline Hospital San Pedro de Alcántara & Público & Hospital para mujeres. \\
\hline Hospital de la Caridad & Público & Hospital para mujeres. \\
\hline Hospital de San Lázaro & Público & $\begin{array}{l}\text { Para los infestados de lepra; el más pobre, fundado a las faldas del río por el espa- } \\
\text { dero Antón Sánchez junto a la Parroquia, en } 1563 \text { a causa de la epidemia de lepra } \\
\text { de ese año. }\end{array}$ \\
\hline Hospital de San Diego & Religioso & $\begin{array}{l}\text { Acoge a los hermanos de San Juan de Dios quienes acuden a éste en su convalecen- } \\
\text { cia, además cumple la función de asilo. }\end{array}$ \\
\hline Hospital de la Buena Muerte & Religioso & $\begin{array}{l}\text { Casa de padres agonizantes fundado en } 1715 \text {. Posteriormente se construyó en el } \\
\text { arrabal de San Lázaro uno similar en } 1736 .\end{array}$ \\
\hline $\begin{array}{l}\text { Hospital de San Cosme y San } \\
\text { Damián }\end{array}$ & Religioso & $\begin{array}{l}\text { Para albergar mujeres enfermas; además, acoge a doncellas pobres. Se encontraba } \\
\text { regentado por las hermanas de la Caridad. }\end{array}$ \\
\hline Hospital de San Juan de Dios & Religioso & Para religiosos convalecientes, ubicado en la zona urbana. \\
\hline Hospital Betlemita "Casa Grande" & Religioso & Ubicado extramuros de la ciudad, para convalecientes. \\
\hline Hospital Betlemita & Religioso & Para enfermos incurables. \\
\hline
\end{tabular}


Por otra parte, la dificultad que representaban los montes de escombros para el rescate de los cadáveres tanto de seres humanos como de caballos, burros y otros animales domésticos que murieron en grandes cantidades, produjo un hedor pestífero que permeaba a la ciudad y que despertó el miedo de que seguirían grandes epidemias. La putrefacción de cadáveres creó condiciones óptimas para las temidas enfermedades, que poco tiempo después del sismo comenzaron a afligir a los sobrevivientes, subiendo la tasa de mortalidad asociada con el desastre. Se desataron epidemias de catarros, enfermedades gastrointestinales y tifoidea, que aumentaron el número de víctimas secundarias, sobre todo entre la numerosa gente que había huido de la ciudad y se había instalado en el campo. Se calculó que murieron más limeños en la zona rural, presa de las enfermedades contagiosas, a la falta de abrigo y a la humedad del campo, que los que fueron sepultados por el terremoto mismo ${ }^{(1)}$.

Los canales y acueductos también se destruyeron, provocando la suspensión del abasto de agua para uso público. El derrumbe de almacenes, panaderías y hornos, junto con la interrupción del transporte, inició un severo periodo de hambre entre los sobrevivientes. La población de Lima contaba con las grandes bodegas del Callao, donde se almacenaban los productos importados que abastecían a la ciudad, sobre todo el trigo, los cebos, el aguardiente, las maderas y los metales como hierro y estaño. Además, los comerciantes que pudieron salvar una parte de sus mercancías de primera necesidad, aprovechando la escasez, las vendían a precios elevados. Otros más comenzaron a sacar ventaja de la miseria, comprando alhajas de plata, oro y piedras preciosas a precios bajísimos a quienes precisaban de dinero para comprar alimentos. También se tuvo que sufrir el saqueo y el robo de los delincuentes que se aprovechaban de la confusión ${ }^{(9)}$.

Sin embargo, las autoridades y, en mayor medida, las cofradías y vecinos prominentes comenzaron muy pronto a enfrentar la situación, buscando medios para luchar contra los problemas que suscitaban el hambre, los heridos, el entiero de los cadáveres y el restablecimiento del orden público.

La necesidad de reconstruir los edificios públicos significó la toma de medidas extraordinarias en cuanto a la recaudación de impuestos. Tal es el caso de la redificación y mejora de los hospitales limeños los cuales sufrieron gran daño tras el terremoto, por lo que el virrey «facilitó arbitrios y trabajó en alentar a los mayordomos y hermandades para el progreso de estas obras importantes» apelando al recurso de las loterías para financiar la reconstrucción de los hospitales de Santa Ana y la Caridad, además de destinar fondos a los hospitales de San Bartolomé y San Andrés ${ }^{(23)}$ de esa forma se fueron restituyendo paulatinamente los servicios sanitarios de la ciudad.

La rápida reacción del virrey Antonio Manso de Velasco evitó el incremento de muertes, ya que ordenó la remoción de escombros y enterramiento masivos de cadáveres en zanjas para prevenir epidemias, la ordenó la limpieza de acueductos, el abastecimiento de alimentos y la reconstrucción de las panaderías. Evitó la especulación de los precios del trigo, prohibiendo su reventa en los caminos. Evitó el saqueo y la delincuencia nombrando alcaldes de barrio cuyas funciones incluían evitar cualquier disturbio, detener a los ladrones, rescatar los cadáveres de las ruinas y darles cristiana sepultura ${ }^{(8)}$. Con estas medidas no solo se buscó retomar el orden interno, sino que también brindó a los sobrevivientes un sentido de seguridad y bienestar.

\section{CONCLUSIONES}

La destrucción de la red hidráulica, la acumulación de escombros y cadáveres, así como las condiciones climáticas posteriores, contribuyeron al incremento de pérdidas humanas, teniendo en cuenta destrucción y daños en los hospitales de Lima y Callao. Junto al brote de epidemias, el impacto negativo en la producción agrícola y abastecimiento de alimentos, el terremoto de 1746 nos permite reconocer el impacto de los desastres naturales en la salud.

Tras el terremoto de 1746 la población recurrió a la religión nuevamente, pero esta vez se produjo un cambio: se pensó en organizar a los vecinos para restablecer el orden, se endurecieron las leyes para prevenir los robos y saqueos, los entierros se realizaban en fosas comunes en las afueras de la ciudad, pero sobre todo se buscó reconstruir la ciudad de forma segura. El virrey Antonio Manso de Velazco, conde de Superunda, tomó parte activa durante el proceso de reconstrucción y le dio un nuevo giro a la intervención y respuesta de las autoridades ante un desastre.

Contribuciones de autoría: Carlos Carcelén, Daniel Morán y Laura Amador han participado en la concepción del artículo, la recolección de datos, su redacción y aprobación de la versión final.

Fuentes de financiamiento: Autofinanciado.

\section{REFERENCIAS BIBLIOGRÁFICAS}

1. Oliver-Smith A. El terremoto de 1746: El modelo colonial, el desarrollo urbano y los peligros naturales. En: García V. (ed.) Historia y desastres en América Latina. Bogotá: Red de Estudios Sociales en prevención de desastres en América Latina. CIESAS; 1997. (2) p.133-161.
2. Petit-Breuilh M. Desastres naturales y ocupación del territorio en Hispanoamérica. Huelva: Universidad de Huelva; 2004.

3. Seiner L. Historia de los sismos en el Perú. Catálogo: Siglos XV-XVII. Lima: Universidad de Lima; 2009. 
4. Walker C. The Upper Classes and Their Upper Stories: Architecture and the Aftermath of the Lima Earthquake of 1746. Hispanic American Historical Review. 2003; 83(1): 53-82.

5. Pérez-Mallaína P. Retrato de una ciudad en crisis: la sociedad limeña ante el movimiento sísmico de 1746. Lima: Pontificia Universidad Católica del Perú - Instituto Riva-Agüero; 2001.

6. Jiménez C. et al. Seismic Source of 1746 Callao Earthquake from Tsunami Numerical Modeling, Journal of Disaster Research. 2013; 8 (2): 270.

7. Pérez-Mallaína P. "Las catástrofes naturales como instrumento de observación social: el caso del terremoto de Lima en 1746”. Anuario de Estudios Americanos. 2005; 62(2): 47-63.

8. Relación que escrive el Conde de Superunda virrey del Perú... La Primera comprehende los años desde 9 de Julio de 1745, hasta fin del mismo més, en el a 1756 y la Segunda desde $1^{\circ}$ de Agosto de dho año hasta 12 de Octubre del de 1761. BNP, C1312.

9. Odriozola M. Terremotos: Colección de las relaciones de terremotos más notables que ha sufrido esta capital y que la han arruinado. Lima: Tipografía de Aurelio Alfaro; 1863.

10. Chuhue Richard. La Piedad Ilustrada y los Necesitados en Lima Borbónica. Una aproximación hacia la plebe limeña y el manejo institucional en el siglo XVIII. Summa Historiæ. 2004; 1 (1).

11. Flores Galindo A. La ciudad sumergida. Aristocracia y plebe en Lima, 1760-1830. Lima: Horizonte; 1991.

12. Manso De Velasco, J. Relación y documentos de gobierno del virrey del Perú, José A. Manso de Velasco, Conde de Superunda (1745-1761). Moreno Cebrián A. (ed.) Madrid: Consejo Superior de Investigaciones Científicas, Instituto “Gonzalo Fernández de Oviedo”; 1983.
13. Lastres J. La medicina en el virreinato. En: Historia de la Medicina Peruana 5(2). Lima: Imprenta Santa María; 1951.

14. Palma R. Anales del Cuzco 1600 a 1750. Lima: Imprenta del Estado; 1901.

15. Figueroa S. Introducción a la Salud Pública [Internet]. Ciudad de Guatemala: Universidad de San Carlos. Facultad de Ciencias Médicas. 2012 [citado el 08 de Noviembre de 2019]. Disponible en: https://saludpublicayepi.files.wordpress.com/2012/06/documento-3er-parcial-compilacion-4-documentos.pdf

16. Navarro V. Concepto actual de la salud pública. En: Martínez F. Salud pública. España. McGraw-Hill Interamericana de España; 1997.p. 49-54.

17. Seaman J, Leivesley S. y Hogg C. Epidemiología de desastres naturales. México D.F: Harla; 1989.

18. Alcedo A. Diccionario geografico-histórico de las Indias Occidentales o America: es a saber: de los Reynos del Peru, Nueva España, Tierra Firme, Chile y Nuevo Reyno de Granada. Vol. 2. Madrid, Imprenta de Blas Román; 1967.

19. Lizárraga R. Descripción breve de toda la tierra del Perú, Tucumán, Río de la Plata y Chile. Libro primero. Lima: Imprenta Americana; 1908.

20. Pérez Cantó, Pilar "La población de Lima en el siglo XVIII", Boletín americanista. 1982; 32: 383-407.

21. Vargas Ugarte, R. Historia del Perú. Virreinato (Siglo XVIII) 1700-1790. Lima: Librería Imprenta Gil; 1956.

22. Reflexiones históricas y políticas sobre el estado de la población de esta capital. Mercurio Peruano, 3 de febrero de 1791. Lima: 92.

23. Mendiburu M. Diccionario histórico biográfico del Perú. Vol. 7. Lima: Librería e imprenta Gil; 1933. 